\title{
Corrected Precision of Topographic Measurements by Radar Interferometry
}

This paper was downloaded from TechRxiv (https://www.techrxiv.org).

\section{LICENSE}

CC BY-SA 4.0

SUBMISSION DATE / POSTED DATE

08-02-2022 / 11-02-2022

CITATION

weissman, isaac (2022): Corrected Precision of Topographic Measurements by Radar Interferometry. TechRxiv. Preprint. https://doi.org/10.36227/techrxiv.19142273.v1

$\mathrm{DOI}$

10.36227/techrxiv.19142273.v1 


\section{Corrected Precision of Topographic Measurements by Radar Interferometry}

ISAAC WEISSMAN, Life Senior Member, IEEE

\begin{abstract}
This communication presents a corrected formulation, differing significantly from a traditional and widelyemployed one, for the precision of topographic measurements by means of space-based or airborne radar interferometry. The discrepancies, which are especially wide at near-nadir viewing, are relevant to a current satellite program.
\end{abstract}

Index Terms-InSAR, interferometry, radar, satellite, topography.

\section{INTRODUCTION}

This letter presents a needed correction to a widely-employed formulation for the precision of estimates of height variations over a surface by means of radar phase interferometry. The latter formulation has been applied to synthetic aperture radar interferometry (InSAR) for topographic measurements from aircraft or space-based platforms, and perpetuated in mainstream technical publications for nearly three decades (e.g., [1][2][3][4][5]).

This communication takes on particular urgency due to its relevance to the interferometer aboard a current satellite program [6] planned for launch later in 2022 [7].

\section{DISCUSSION}

For convenience, we refer here to the notation and formulation of [1]. Fig. 1 is a depiction of the pertinent geometry, with an interferometer located at an altitude $h$, and radar antennas (A1 and A2) located at opposite ends of the interferometer baseline, of length $B$. In this notation, $r$ is the nominal range to a topographic feature, $\delta$ is the difference in range between the two paths of the interferometer, $\Theta$ is the offnadir view angle, and $\alpha$ is the tilt angle of the baseline upward from the horizontal. The height of a feature, $z$, at a horizontal distance $y$ is measured relative to a horizontal plane.

It is important to observe that, in Fig. 1, a change in $\mathrm{z}$ causes a change in $r$ as well as in $\Theta$ at a given horizontal location.

Note that the known earth's curvature can easily be taken into account and is not important for purposes of the current discussion.

The difference, $\varnothing$, between the phases of the signals received at the antennas is used to measure $z$.

The author is an independent radar consultant based in Washington, DC, 20007, USA (e-mail: weissman.ike@ieee.org).
In the "traditional" formulations (each in their own notation), the relationship between the error in height estimate, $\sigma_{z}$, and the error, $\sigma_{\varnothing}$, in the measurement of phase difference is given, approximately, in [1] as

$$
\begin{gathered}
\sigma_{z} \quad \lambda r \sin \Theta \\
-\sigma_{\varnothing} \quad 2 \pi k B \cos (\Theta-\alpha)
\end{gathered}
$$

In this expression, $\lambda$ is the radar wavelength, and $k$ is 1 or 2 depending, respectively, on whether the radar transmissions are from one or both antennas.

Two comments are here offered in connection with (1). The first is that it is not restricted to the moderate view angles typical of InSAR. Indeed, the same expression is invoked for nearnadir viewing planned for the aforementioned interferometer in a current satellite program [6].

The second comment is that the expression predicts a perfect height estimate for nadir viewing $(\Theta=0)$, regardless of the quality of the phase difference measurements or the length of the baseline. Yet, a simple physical model indicates quite the opposite, as shown in Fig. 2.

The physical model assumes a horizontal baseline $(\alpha=0)$ which results in identical lengths for the two paths of the interferometer. It is clear that the phase difference received at the antennas is essentially insensitive to the height of the measured surface feature along the vertical dashed line. This indicates that something is apparently wrong in (1).

The apparently-incorrect formulation arises as follows: In Fig. 1,

$$
z=h-r \cos \Theta
$$

Reference [1], in order to express $d z / d \varnothing$, differentiates $z$ with respect to $\Theta$, but fails to differentiate $z$ with respect to $r$.

A corrected expression is offered here, based on Fig. 1: By forming right triangles, we obtain the difference in interferometer path lengths as

$$
\begin{aligned}
\delta= & (r+\delta)-r=\left[(h-z-B \sin \alpha)^{2}+y^{2}\right]^{1 / 2} \\
& -\left[(h-z)^{2}+(y-B \cos \alpha)^{2}\right]^{1 / 2}
\end{aligned}
$$

Expression (3) may appear somewhat complicated, but at least it is exact.

The relationship between $\delta$ and phase difference, $\varnothing$, must take into account the phase ambiguities (modulo $2 \pi$ ). The relationship is, then,

$$
\varnothing+2 \pi n=2 \pi k \delta / \lambda
$$

where $n$ is an integer. The uncertainty in the proper value of $n$ causes topographic measurement ambiguities (akin to grating lobes in phased arrays) which require phase unwrapping, a topic widely discussed in technical publications. Fortuitously, in this communication we are concerned only in the measurement precision, irrespective of the unwrapping; that is, 


$$
d \delta=(\lambda / 2 \pi k) d \varnothing
$$

The corrected precision of the topographic measurements can then be found by taking derivatives and using (4):

$$
\frac{\sigma_{z}}{-\sigma_{\varnothing}} \approx \frac{d z}{d \varnothing}=\frac{d z}{d \delta} \cdot \frac{d \delta}{d \varnothing}=\frac{1}{(d \delta / d z)} \cdot \frac{\lambda}{2 \pi k} .
$$

The key term ( $d \delta / d z)$ is found by differentiating (3) and is given in the Appendix.

Fig. 3 shows examples of the precision of topographic height estimates at X-band $(\lambda=0.032 \mathrm{~m})$ vs. distance, $y$, along the horizontal plane for representative interferometer parameters and two values of baseline tilt angle, $\alpha$. Also shown are the estimates using the traditional formulation (1), wherein the angle, $\theta$, and nominal range, $r$, are expressed in terms of $y$. Both formulations assume radar transmissions from both antennas $(k$ $=2$ ), as is the case in [1].

The discrepancies in Fig. 3 between the formulations are striking, especially for near-nadir viewing. The corrected results are consistent with the simple physical model of Fig. 2 and indicate poor measurement precision at small view angles.

These smaller view angles are of particular interest for certain ocean and land topographic measurements, as in the aforementioned satellite program. A special case examined is then the case of a downlooking interferometer with a horizontal baseline $(\alpha=0)$. Using (8) in the Appendix and inserting in (5), we then obtain

$$
\left.\frac{\sigma_{z}}{\sigma_{\varnothing}} \approx \frac{\lambda}{(2 \pi k)} \quad \frac{h}{\left[(y-B)^{2}+h^{2}\right]^{1 / 2}}-\frac{h}{\left(y^{2}+h^{2}\right)^{1 / 2}}\right\}^{-1}
$$

We note, in (6), that as $y \rightarrow B / 2$ (directly underneath the interferometer) $\sigma_{z} / \sigma_{\varnothing} \rightarrow \infty$, which is consistent with the interpretation of the physical model of Fig. 2.

Surface height measurements near nadir are normally conducted by radar altimetry as described, for example, in [8], using wideband range measurements, and not by phase interferometry.

\section{CONCLUSIONS}

Substantial discrepancies exist between the commonly cited (traditional) formulation of the precision of topographic measurements by radar interferometry and the alternative formulation offered here.

The discrepancies are particularly significant with regard to topographic measurements at small off-nadir viewing angles, where the corrected formulation predicts severely degraded precision.

It can be concluded that phase interferometry is apparently unsuited for near-nadir radar topography.

Instead of phase interferometry, for near-nadir angles, it may be advisable to employ a technique akin to a Michelson Interferometer. This would allow very precise range measurements to the ocean surface by transmitting, in a pair of wideband pulses with separate frequencies, and observing the vector sum of their received echoes. If this technique were to be employed, at least two challenges would remain: (a) phase "unwrapping" and (b) beamwidth "smearing." The unwrapping could be performed by transmitting a third pulse whose frequency departs only slightly from one of the others. The smearing could be mitigated by employing a sufficiently wide single antenna aperture consisting of a phased array, perhaps using the exacting design of [9] for the individual array elements. In addition, wideband individual pulses (e.g., with $10 \%$ bandwidth) could assist by isolating different portions of the beam projection on the surface.

A simpler alternative approach would only employ wideband ranging. For example, the use of a $10 \%$-bandwidth pulse centered at $\mathrm{K}_{\mathrm{a}}$-band could provide a precision of several centimeters with large enough signal-to-noise ratios.

\section{APPENDIX}

For (3), the derivative of $\delta$ with respect to $Z$ is found by direct differentiation:

$$
\begin{array}{lcc}
d \delta & h & (h-B \sin \alpha) \\
\hdashline d z & \approx\left[(y-B \cos \alpha)^{2}+h^{2}\right]^{1 / 2} & - \\
\left.\hdashline y^{2}+(h-B \sin \alpha)^{2}\right]^{1 / 2}
\end{array}
$$

in which the approximation $h \approx(h-z)$ is included since, generally, $h>>$.

This expression is consistent with numerical differentials taken in the "corrected" examples of Fig. 3.

For the special case of a horizontal baseline $(\alpha=0)$,

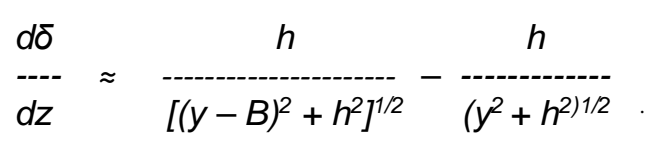

\section{REFERENCES}

[1] H. A. Zebker, C. L. Werner, P. A. Rosen, and S. Hensley, "Accuracy of topographic maps derived from ERS-1 interferometric radar," IEEE Trans. Geoscience and Remote Sensing, vol. 32, no. 4, p. 824, July 1994

[2] A. Moreira, P. Prats-Iraola, M. Younis, G. Krieger, I. Hajnsek, and K. P. Papathanassiou, "A tutorial on synthetic aperture radar," IEEE Geoscience and Remote Sensing Magazine, vol. 1, p. 22, Mar. 2013

[3] M. A. Richards, "A beginner's guide to interferometric SAR concepts and signal processing," IEEE A\&E Systems Magazine, vol. 21, no. 6, p. 7, June 2006

[4] R. J. Sullivan, "Synthetic Aperture Radar," in Radar Foundations for Imaging and Advanced Concepts, Raleigh, NC, USA, SciTech Publishing, 2004, p. 227

[5] P. A. Rosen, S. Hensley, I. R. Joughin, F. K. Li, S. N. Madsen, E. Rodriguez, and R. M. Goldstein, "Synthetic aperture radar interferometry," Proc. IEEE, vol. 88, no. 3, p. 343, Mar. 2000

[6] L. Fu, D. Alsdorf, R. Morrow, E. Rodriguez, N. Mognard, "SWOT: The Surface Water and Ocean Topography Mission," 
Jet Propulsion Laboratory, Pasadena, CA, USA, pp. 147-148, JPL Publication 12-05, Feb. 2012

[7] Jet Propulsion Laboratoy. (2021, Dec. 13) NASA to Launch 4 Earth Science Missions in 2022, https://swot.jpl.nasa.gov

[8] R. K. Raney, "Space-Based Remote Sensing Radars," in Radar Handbook, 3rd ed., USA, McGraw-Hill, 2008, pp. 18.2918.42

[9] R. E. Hodges, et al, "An extremely large $\mathrm{K}_{\mathrm{a}}$-band reflectarray antenna for interferometric synthetic aperture radar,” IEEE A\&P Magazine, pp. 23-33, Dec. 2020

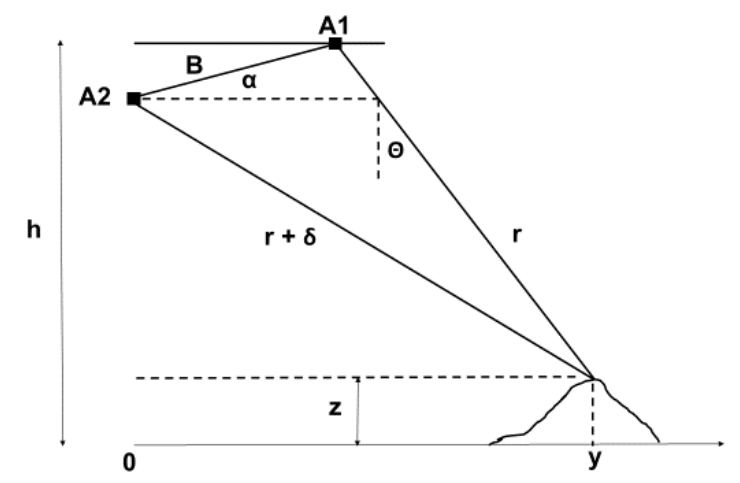

FIGURE 1. Reference [1] interferometer geometry.

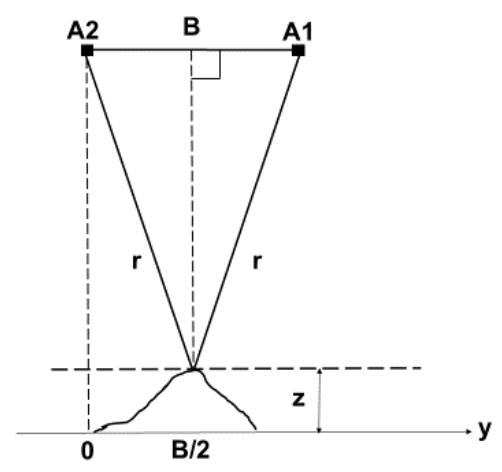

FIGURE 2. Illustrative physical model.

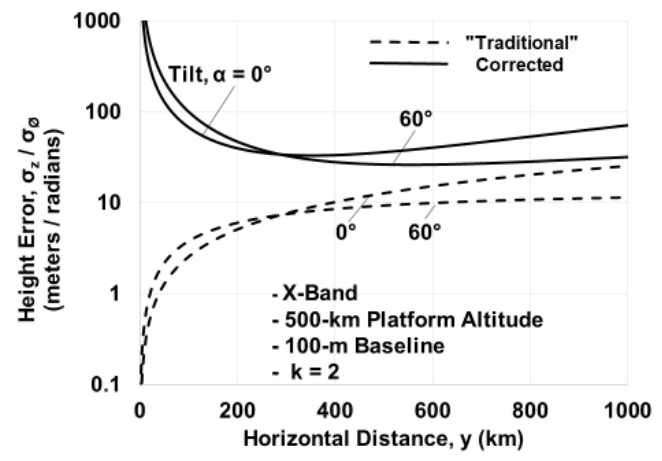

FIGURE 3. Comparison of topographic measurement precision vs. horizontal distance. 